\title{
NEAR-FIELD ELECTROMAGNETIC MODELING AND ANALYSIS
}

C. Long Yu*

Naval Air Warfare Center Weapons Division, Point Mugu, CA 93042

R. Kipp, D. J. Andersh and S. W. Lee DEMACO Inc., Champaign IL 61820

1.0 Introduction: For missile fuzing applications, the transmitting and receiving antennas on a missile are generally located in the near-field zone of the scattered field from the target encountered. In consequence, the radar return computation is complicated by the partial target illumination, nonuniform antenna patterns, target material coatings, and engine inlet returns. Existing near-field radar cross section (RCS) computation algorithms, such as NcPTD [1], are typically based on first-order high-frequency methods that do not account for multiple bounce, complex shadow effects and scattering from a cavity, such as an engine inlet or a sensor box. A successful missile/target engagement analysis must thus be conducted with accurate near-ficld target RCS information that includes effects attributable to multi-bounce, complex-shape shadowing, material coatings, antenna patterns, etc.. This paper discusses techniques for solving near-field modeling problems in missile/target end-game scenarios.

2.0 Technical Approach: In an encounter as shown in Fig. 1, the missile passes from the far-field scattering zone, through the intermediate-zone and into the near-zone of the target. Most radar scattering predictors make the assumption that the target is in the farfield of the radar. The far-field assumption is that the wavefront incident at the target has uniform magnitude and phase, and the scattered wave arrives back at the radar antenna from a single direction, as shown in Figure 1. In the near-zone, however, the missile and target are in close proximity, and an EM wavefront of nonuniform magnitude and phase dictates the complete scattering phenomena. So, far-field assumptions lead to gross errors under near-field conditions.
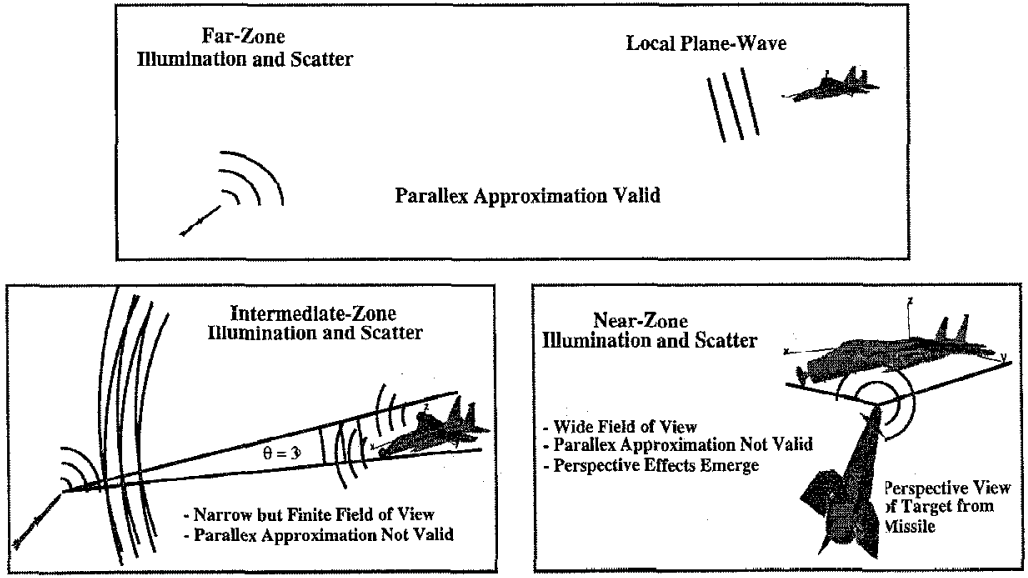

Figure 1. Definition of far-zone, intermediate-zone, and near-zone for a missile encounter with a target.

To solve the near-field problems in missile/target end-game scenarios, we chose a highfrequency approximation, shooting and bounce rays (SBR) method, as the core EM computational engine for our Npatch modeling development. SBR uses geometrical

\section{U.S. Government Work Not Protected by U.S. Copyright}


optics (GO) ray tracing to implement physical optics (PO) [2-3] and is suitable for electrically large and geometrically complex targets represented by CAD models in nearzone missile/target encounters where the target spans a range of aspect angles with respect to the missile. SBR has the following advantages and features: a) SBR gracefully handles highly complex and realistic CAD models, b) includes multi-bounce scattering mechanisms, c) easily handles surfaces coated with layers of materials, and d) inherently includes PO diffraction effects for both metallic and coated edges. Npatch uses 3-D ray tracing on CAD models composed of triangular facets to implement a PO/SBR scattering solution. It includes the radiation and receiving patterns of the missile fuzing and tracking antennas. Npatch has significant capability and accuracy enhancements over NcPTD which is a single-bounce PO code for simple geometries defined by plates, cylinders, curved surfaces, cones, etc. NcPTD contains no ray tracer for accurate determination of complex shadowing and has no mechanism for including multi-bounce effects in complex 3-D target models.

The modeling process of the Npatch code is illustrated in Figure 2. A missile is located at point $\mathbf{R}_{\mathbf{m}}$ and has an instantaneous velocity $\boldsymbol{v}_{\mathbf{m}}$; its target is located at point $\mathbf{R}_{\mathbf{t}}$ with velocity $v_{\mathbf{t}}$. The antenna (located at $\mathbf{R}_{\mathbf{a}}$ ), as mounted on the missile, has a radiation pattern $\mathbf{A}\left(\theta_{\mathrm{m}}, \phi_{\mathrm{m}}\right)$. This antenna illuminates the target, which scatters energy in all directions. Some of that energy scatters back toward the missile and is partially absorbed by the antenna. The quantity of interest is the ratio of the received power to the transmitted power $\mathrm{P}_{\mathrm{r}} / \mathrm{P}_{\mathrm{t}}$ at the antenna terminals, including the phase shift, as a function of frequency. The SBR method is applied to compute this quantity in the following manner.
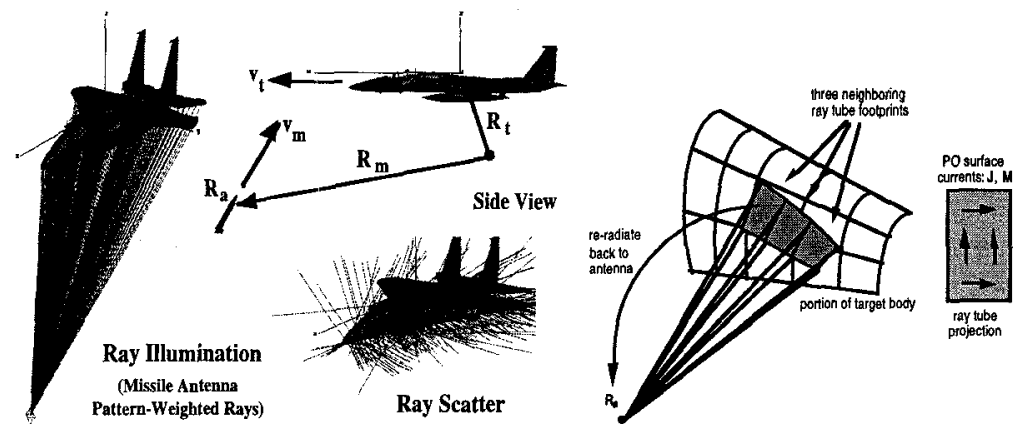

Figure 2. End-game encounter: Antenna patternweighted rays are launched from missile and scatter off of the target, leaving surface currents that radiate back to the missile.

Figure 3. Rays launched from missile antenna project ray tubes on to target surface.

First, the target is illuminated by thousands of rays weighted by the radiation pattern of a mounted missile antenna. These rays are launched from the missile antenna as if emanating from a point source toward the target (see Fig. 2). A CAD ray-tracer is then used to determine which target surfaces are lit and which surfaces are shadowed. Hence, the cast shadow problem is properly handled in the SBR process. Second, the illuminating rays are treated as ray tubes, which cast "footprints" on the target body as shown for several rays in Figure 3. Using physical optics (PO) principles, the induced surface currents over the domain of each footprint are computed. This is where the material properties of the target are incorporated; the computed surface currents depend on the material attribute of the surface at each ray hit point. Using free-space Green's function, we compute the scattered energy at the point $\mathbf{R}_{\mathbf{a}}$ from each footprint. Only a fraction of this energy will be absorbed by the missile antenna, and this will depend on the direction of arrival. Hence, in computing $\mathrm{P}_{\mathbf{r}} / \mathrm{P}_{\mathbf{t}}$, we weight the scattered energy at $\mathbf{R}_{\mathbf{a}}$ 
by the receiving cross-section of the missile antenna at the arrival angle. Finally, the rays which illuminate target surfaces are specularly reflected from their hit points. The ray tracer continues to trace these rays until they escape. Some will escape after the first bounce, and they produce no further contributions at the receiver. This process produces the 1 st-bounce contribution similar to NcPTD. Others will become multi-bounce rays, also shown in Figure 2. These rays continue to induce further currents on the target surface, which are then radiated back to the missile antenna in the same manner as described above. As a result, they also contribute to the radar received power. In this sense, SBR can produce much more accurate near-field scattering results than any available near-field predictors.

3.0 Simulation Results: To demonstrate the capabilities and validity of the newly developed Npatch scattering predictor, we have applied it to a variety of simple and complex 3-D targets in end-game (near-field) encounters with a missile. Experimental measurements conducted at Naval Air Warfare Center Weapons Division for end-game encounter testing involving many different targets and fuzes are used to validate our nearfield modeling simulations. We also compare the Npatch single bounce and multi-bounce predictions on a more complex multi-bounce geometry to show the extra contributions that a multi-bounce implementation can generate. Npatch is first applied to a solid, metallic cylinder in a end-game simulation. The arrangement for the end-game encounter is shown in Figure 4 for a missile moving on a linear track under a solid, metal cylinder. The missile fixture has a 14-element array whose main beam points $10^{\circ}$ forward of broadside. Experimental measurements conducted by the NAWCWPNS was used to validate the single bounce missile/target code, NcPTD and the Npatch code. As can be seen in Figure 4, the computed results compared very favorably with the measured data and NcPTD computation. Npatch is next applied to a new target, an almond. This is to demonstrate its capability in handling near-field scattering returns, and material coating. The comparison is shown in Figure 5. The results agree well with results obtained from NcPTD. Finally, the new code is applied to a perfectly conducting MIG-29 model which is the sort of realistic CAD model geometry that goes beyond the capabilities of codes like NcPTD. The MIG-29 model offers ample opportunities for multi-bounce scattering mechanisms, and this is evidenced in the Npatch predicted scattering returns shown in Figure 6. For the multi-bounce trace, Npatch is directed to trace rays up to 50 bounces. Clearly, much of the scattering response for this combination of CAD model and missile path comes from indirectly illuminates surfaces - that is, surfaces illuminated by energy reflected off other parts of the aircraft body. The antenna pattern is identical to the one described in Figure 4.
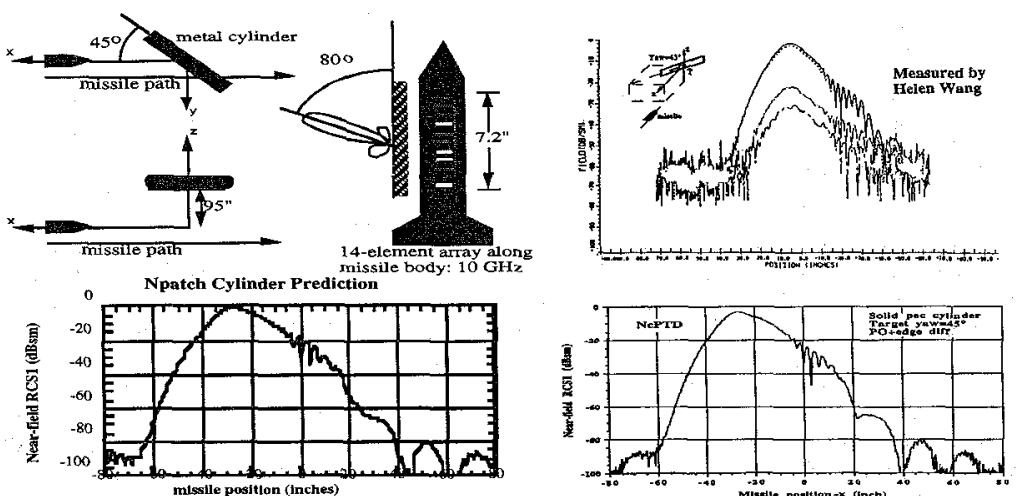

Figure 4. Near-field RCS results of a cylinder versus missile position: measurement, NcPTD prediction, and Npatch prediction. 


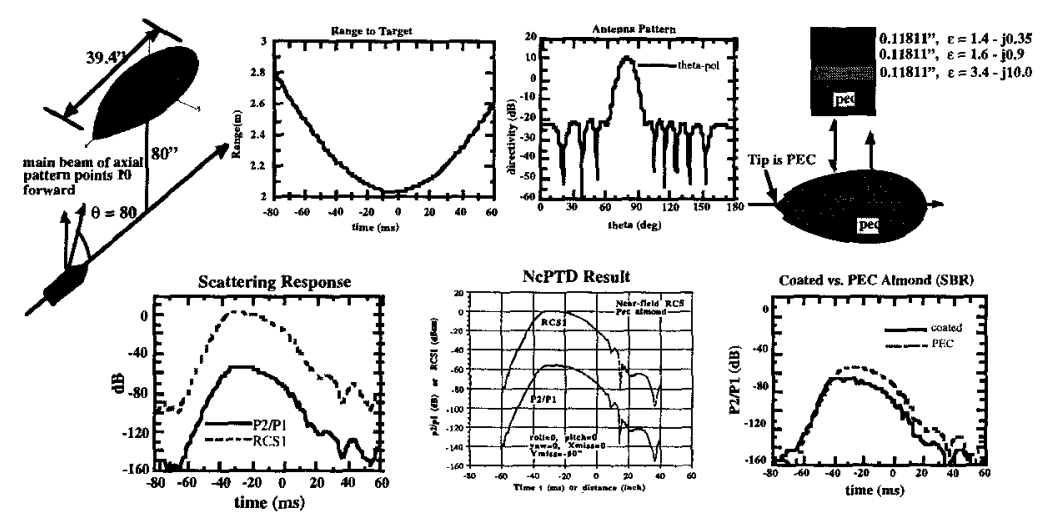

Figure 5. Almond (PEC) results demonstrating near-field scattering response and material coating effect.

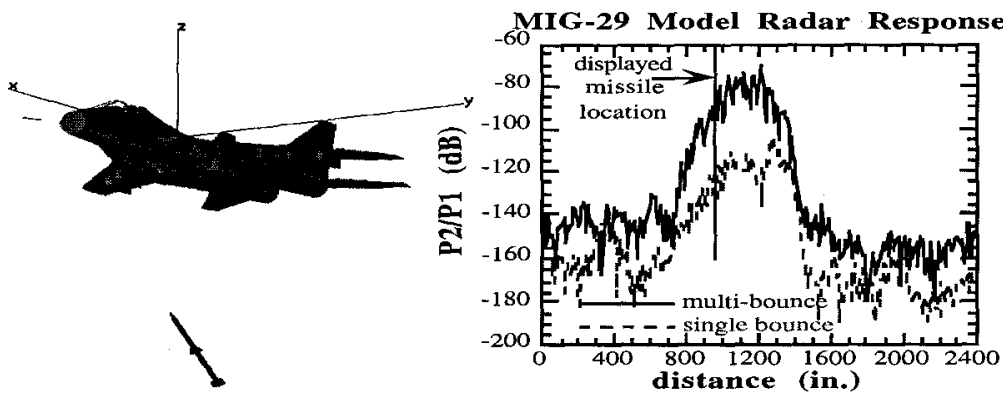

Figure 6. Significance of multi-bounce in MIG-29 model scattering return predicted by Npatch at $10 \mathrm{GHz}$.

4.0 Conclusion: As a result of our effort to improve radar-target scattering modeling capability for endgame simulation, Npatch was developed. Npatch is a deterministic, multi-bounce EM scattering model which simulate the radar returns of an airborne target from mid-course to endgame. Features include non-uniform target illumination by arbitrary missile antenna patterns (and non-uniform scattering reception), mono-static and bi-static modes, multi-bounce scattering, and support for realistic 3-D targets with material coatings. More specifically, we have developed a prototype deterministic nearfield scattering predictor that has now been demonstrated and incrementally validated.

\subsection{References:}

[1] S. W. Lee and S. K. Jeng, NcPTD - 1.2: A High Frequency Near-field RCS Computation Code Based on Physical Theory of Diffraction, DEMACO, Inc., Champaign, IL, 1991.

[2] H. Ling, R. Chou, and S. W. Lee, "Shooting and bouncing rays: calculating the RCS of an arbitrarily shaped cavity," IEEE Trans. Antennas Propagat., vol. 37, 1988, pp. 194-205.

[3] D.J. Andersh, S.W. Lee, et al., "XPATCH: a high frequency electromagnetic scattering prediction code and environment for complex three-dimensional objects," IEEE Antennas and Propagation Magazine, vol. 36, no. 1, Feb. 1994, pp. 65 - 69. 